\title{
Z Boson Decay into Light and Darkness
}

\author{
M. Fabbrichesi, ${ }^{1}$ E. Gabrielli, ${ }^{2,3}$ and B. Mele ${ }^{4}$ \\ ${ }^{1}$ INFN, Sezione di Trieste, Via Valerio 2, 34127 Trieste, Italy \\ ${ }^{2}$ Physics Department, University of Trieste, Trieste, Italy \\ ${ }^{3}$ NICPB, Rävala 10, Tallinn 10143, Estonia \\ ${ }^{4}$ INFN, Sezione di Roma, P.le Aldo Moro 2, 00185 Roma, Italy
}

(Received 20 December 2017; published 26 April 2018)

\begin{abstract}
We study the $Z \rightarrow \gamma \bar{\gamma}$ process in which the $Z$ boson decays into a photon $\gamma$ and a massless dark photon $\bar{\gamma}$, when the latter couples to standard-model fermions via dipole moments. This is a simple yet nontrivial example of how the Landau-Yang theorem-ruling out the decay of a massive spin-1 particle into two photons-is evaded if the final particles can be distinguished. The striking signature of this process is a resonant monochromatic single photon in the Z-boson center of mass together with missing momentum. LEP experimental bounds allow a branching ratio up to about $10^{-6}$ for such a decay. In a simplified model of the dark sector, the dark-photon dipole moments arise from one-loop exchange of heavy dark fermions and scalar messengers. The corresponding prediction for the rare $Z \rightarrow \gamma \bar{\gamma}$ decay width can be explored with the large samples of $Z$ bosons foreseen at future colliders.
\end{abstract}

DOI: $10.1103 /$ PhysRevLett.120.171803

Consider the decay of a massive spin-one particle into two massless spin-one particles. At first glance, this channel should vanish - as it does in the case of two final photons-as dictated by the Landau-Yang theorem [1]. Yet the theorem need not apply if the two final states can be distinguished. This is the case when the final state is made of a photon $\gamma$ and a dark photon $\bar{\gamma}$.

The possibility of extra $U(1)$ gauge groups-with dark photons mediating interactions among the dark-sector particles, which are uncharged under the standard-model (SM) gauge groups-is the subject of many theoretical speculations and experimental searches (see Ref. [2] for recent reviews, mostly for the massive case).

The case of massless dark photons is perhaps the most interesting because the dark photon can be completely decoupled from the SM [3], and interactions between SM fermions and dark photons take place only by means of higher-order operators [4], which are automatically suppressed. Possible experimental tests of this scenario have been investigated in Higgs physics [5], flavor-changing neutral currents [6], and kaon physics [7]. Its relevance for dark-matter dynamics has been discussed in Ref. [8].

The decay of a $Z$ boson into one SM and one dark photon would be a most striking signature for both the existence of dark photons, and the embodiment of the nonapplicability

Published by the American Physical Society under the terms of the Creative Commons Attribution 4.0 International license. Further distribution of this work must maintain attribution to the author(s) and the published article's title, journal citation, and DOI. Funded by SCOAP ${ }^{3}$. of the Landau-Yang theorem. The process can proceed at one loop via SM-fermion exchange. To bypass the theorem, the photon and dark photon must couple differently to the fermions in the loop so as to be distinguishable. This naturally occurs for massless dark photons since they do not have a Dirac (i.e., mediated by a single $\gamma$ matrix) interaction but only a Pauli (i.e., mediated by two $\gamma$ matrices) dipole interaction:

$$
\mathcal{L} \sim \bar{\psi} \sigma_{\mu \nu}\left(d_{M}+i \gamma_{5} d_{E}\right) \psi B^{\mu \nu}
$$

where $B_{\mu \nu}$ is the field strength associated with the dark photon field $B_{\mu}$, and $\sigma_{\mu \nu}=1 / 2\left[\gamma_{\mu}, \gamma_{\nu}\right]$.

For massive dark photons, $z^{\prime}$, the leading interaction would be of the same SM-photon Dirac type as the photon, $e \bar{\psi} \gamma \gamma_{\mu} \psi B^{\mu}$, decreased by the mixing parameter $\epsilon$ [2]. The $Z \rightarrow \gamma z^{\prime}$ channel would then be doubly suppressed by an $\epsilon^{2}$ factor and an extra term $\mathcal{O}\left(m_{z^{\prime}}^{2} / M_{Z}^{2}\right)$, which brings back the outcome of the Landau-Yang theorem for $m_{z^{\prime}} \rightarrow 0$. In this case too then, the higher-order Pauli dipole interaction might be the most relevant, as it is in the case of a massless dark photon. The following analysis can then be extended in a straightforward way to the massive dark-photon case.

The experimental signature for $Z \rightarrow \gamma \bar{\gamma}$ is quite simple and distinctive. In the $Z$-boson center-of-mass frame, the photon is monochromatic with an energy of about $45 \mathrm{GeV}$. A massless dark photon has a neutrinolike signature in a typical experiment [5], and appears as missing momentum in the $Z \rightarrow \gamma+X$ final state. Such a process has been explored at LEP (in the assumption of $X$ being either a $\nu \bar{\nu}$ pair or a hypothetical axion, if sufficiently light) to find the 
limit of $10^{-6}$ (95\% C.L.) for the corresponding branching ratio (BR) [9].

Effective dipole moments in a simplified model of the dark sector.-We compute the dipole operators of Eq. (1) in a simplified-model framework, where we make as few assumptions as possible on the structure of the dark sector.

We extend the SM field content by a new (heavy) dark fermion $Q$, which is a singlet under the SM gauge interactions, but is charged under the unbroken $U_{D}(1)$ gauge group associated to the massless dark photon. We focus on the up-quark kind of interaction. The dark fermion couples to SM fermions by means of a Yukawa-like interaction given by

$$
\mathcal{L} \supset g_{L}^{f}\left(\bar{Q}_{L} q_{R}^{f}\right) S_{R}+g_{R}^{f}\left(\bar{Q}_{R} q_{L}^{f}\right) S_{L}+\text { H.c. },
$$

where $S_{L}$ and $S_{R}$ are new (heavy) messenger scalar particles, and $S_{L}$ is an $S U(2)$ doublet. In Eq. (2), $q_{L}^{f}$ and $q_{R}^{f}$ stand for SM fermions of flavor $f$-that is, $S U(3)$ triplets and, respectively, $S U(2)$ doublets and singlets. The $S_{L}$ messenger field is a $S U(2)$ doublet, $S_{R}$ is a $S U(2)$ singlet, and both are $S U(3)$ color triplets (singlets) for quark (lepton) messengers. Both fields are also charged under $U_{D}(1)$, carrying the same dark-fermion charge.

In order to generate chirality-changing processes, the mixing terms of the kind (see Ref. [10] for more details)

$$
\mathcal{L} \supset \lambda_{S} S_{0}\left(\tilde{H}^{\dagger} S_{L} S_{R}^{\dagger}+S_{L}^{\dagger} S_{R} H\right)
$$

are required, where $H$ is the SM Higgs boson, $\tilde{H}=i \sigma_{2} H^{*}$, and $S_{0}$ a scalar singlet. After both $S_{0}$ and $H$ take a vacuum expectation value (VEV) $\left(\mu_{S}\right.$ and $v$-the electroweak VEV - respectively), the Lagrangian in Eq. (3) gives rise to the mixing.

Then, each of the messenger fields $S_{ \pm}$(obtained from $S_{L, R}$ by diagonalization) couples to both left- and righthanded SM fermions of flavor $f$ with strength $g_{L}^{f} / \sqrt{2}$ and $g_{R}^{f} / \sqrt{2}$, respectively. We can assume that the size of the mixing [proportional to the product of the VEVs $\left(\mu_{s} v\right)$ ] is large and of the same order of the heavy-fermion and heavy-scalar masses.

The resulting model can be considered as a template for many models of the dark sector, and is a simplified version of the model in Ref. [10], which might provide a natural solution to the SM flavor-hierarchy problem.

The SM Lagrangian plus the terms in Eqs. (2)-(3) and the corresponding kinetic terms provides a simplified model for the dark sector and the effective interaction of a massless dark photon $\bar{\gamma}$ with the SM fields. Then, SM fermions couple to $\bar{\gamma}$ only via non-renormalizable interactions [4] induced by loops of dark-sector particles. The corresponding effective Lagrangian is equal to

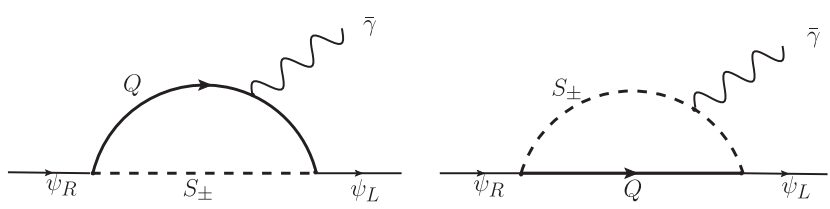

FIG. 1. One-loop vertex diagrams giving rise to the effective dipole operators in Eq. (4) between SM fermions and the dark photon $\bar{\gamma}$. Dark-sector fermions $(Q)$ and scalars $\left(S_{ \pm}\right)$run inside the loop. $\psi_{L}$ and $\psi_{R}$ are SM chiral fermions of arbitrary flavor.

$$
\mathcal{L}=\sum_{f} \frac{e_{D}}{2 \Lambda} \bar{\psi}_{f} \sigma_{\mu \nu}\left(d_{M}^{f}+i \gamma_{5} d_{E}^{f}\right) \psi_{f} B^{\mu \nu}
$$

where the sum runs over all the SM fields, $e_{D}$ is the $U_{D}(1)$ dark elementary charge (we assume universal couplings), $\Lambda$ the effective scale of the dark sector, $\psi_{f}$ a generic SM fermion field. The magnetic and electric dipoles are given by

$$
d_{M}^{f}=\frac{1}{2} \operatorname{Re} \frac{g_{L}^{f} g_{R}^{f *}}{(4 \pi)^{2}} \quad \text { and } \quad d_{E}^{f}=\frac{1}{2} \operatorname{Im} \frac{g_{L}^{f} g_{R}^{f *}}{(4 \pi)^{2}}
$$

respectively.

The operators in Eq. (4) arise via one-loop diagrams after integrating out the heavy dark-sector states (see Fig. 1). Two mass parameters are relevant in the integration: the dark-fermion mass $M_{Q}$, parametrizing chiral-symmetry breaking in the dark sector, and the mass of the lightestmessenger $m_{S}$. As far as the contribution to the magneticdipole operator (with vanishing quark masses) is concerned, for $m_{S} \gg M_{Q}$ one has a chiral suppression, with a $M_{Q} / m_{S}^{2}$ scaling, while for $m_{S} \ll M_{Q}$ one has a $1 / M_{Q}$ behavior, due to the decoupling built in the theory. In order to reduce the number of dimensionful parameters, we have introduced in Eq. (4) a dark-sector effective scale $\Lambda$, defined as the common mass of the dark fermion and the lightest messenger scalar. This choice corresponds to the maximal chiral enhancement. Nevertheless, because the dipole moment in Eq. (4) is proportional to the messenger mixing [see Eq. (3) and following text], the effective scale $1 / \Lambda$ is also proportional to the ratio $v \mu_{S} / m_{S}^{2}$, as expected from the $S U(2)$ symmetry breaking. Since we are assuming a large-mixing scenario, $v \mu_{S} / m_{S}^{2}$ is of order 1 , and one can express the effective scale as in Eq. (4) with $\Lambda \sim m_{S} \sim M_{Q}$.

Stringent limits on the scale and couplings of the dark sector come from flavor physics [6,7] and astrophysics [11]. In order to evade them, we restrict ourselves to flavor diagonal interactions of heavier quarks and leptons for which there are currently no bounds.

Amplitudes.-We are interested in the decay process of a $Z$ boson into two massless spin-one particles: 


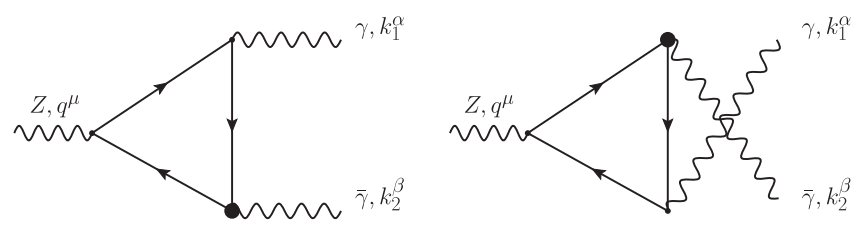

FIG. 2. Feynman diagrams for the decay $Z(q) \rightarrow \gamma\left(k_{1}\right) \bar{\gamma}\left(k_{2}\right)$. The blob represents the insertion of the dipole operator in Eq. (4). The case of two photons with the same interaction (no blobs) would lead to a cancellation as dictated by the Landau-Yang theorem.

$$
Z(q) \rightarrow \gamma\left(k_{1}\right) \bar{\gamma}\left(k_{2}\right)
$$

where $k_{1}$ and $k_{2}$ are the photon and dark-photon 4-momenta, respectively, and $q=k_{1}+k_{2}$ is the Z-boson 4-momentum. The total amplitude $\mathcal{M}$ for the decay process is obtained by computing the one-loop diagrams represented in Fig. 2. It is given by

$$
\mathcal{M}=\mathcal{M}_{M}+\mathcal{M}_{E}
$$

where the $\mathcal{M}_{M}$ and $\mathcal{M}_{E}$ stand for the magnetic- and electric-dipole moment contributions.

In both amplitudes in Eq. (7) the ultraviolet divergencies cancel out in the sum of the two diagrams in Fig. 2, and the result is finite. We use dimensional regularization. The $\gamma_{5}$ matrix can be treated naively as anticommuting with all other $\gamma$ matrices as long as the final expression is fixed by requiring that the Ward identities are satisfied [12]. All terms are proportional to the fermion masses and no gauge anomaly is involved.

The $C P$-conserving part of the amplitude is given by

$$
\mathcal{M}_{M}=\frac{e_{D} e}{4 \pi^{2}} \sum_{i=1}^{3} C_{i} Q_{i}^{\mu \alpha \beta} \epsilon_{\mu}^{Z}(q) \epsilon_{\alpha}\left(k_{1}\right) \bar{\epsilon}_{\beta}\left(k_{2}\right)
$$

where $e$ is the electric charge, $\epsilon_{\mu}^{Z}(q), \epsilon_{\alpha}\left(k_{1}\right), \epsilon_{\beta}\left(k_{2}\right)$ are the $Z, \gamma$, and $\bar{\gamma}$ polarization vectors, respectively, and

$$
\begin{gathered}
Q_{1}^{\mu \alpha \beta}=\varepsilon^{\alpha \beta \mu \lambda} k_{1 \lambda}-\frac{2}{M_{Z}^{2}} \varepsilon^{\alpha \mu \lambda \rho} k_{1}^{\beta} k_{1 \lambda} k_{2 \rho}, \\
Q_{2}^{\mu \alpha \beta}=\varepsilon^{\alpha \beta \mu \lambda} k_{2 \lambda}-\frac{2}{M_{Z}^{2}} \varepsilon^{\beta \mu \lambda \rho} k_{2}^{\alpha} k_{1 \lambda} k_{2 \rho}, \\
Q_{3}^{\mu \alpha \beta}=\frac{1}{M_{Z}^{2}}\left(k_{1}^{\mu}-k_{2}^{\mu}\right) \varepsilon^{\alpha \beta \lambda \rho} k_{1 \lambda} k_{2 \rho},
\end{gathered}
$$

are gauge invariant operators. The coefficients $C_{i}$ are given by

$$
\begin{aligned}
C_{1} & =\sum_{f} \frac{r_{f} m_{f} d_{M}^{f}}{\Lambda}\left(2+B_{f}+2 C_{f} M_{Z}^{2}\right), \\
C_{2} & =\sum_{f} \frac{r_{f} m_{f} d_{M}^{f}}{\Lambda}\left(3+2 B_{f}-2 C_{f} m_{f}^{2}\right), \\
C_{3} & =\sum_{f} \frac{r_{f} m_{f} d_{M}^{f}}{\Lambda}\left(11+5 B_{f}+2 C_{f}\left(m_{f}^{2}+M_{Z}^{2}\right)\right),
\end{aligned}
$$

where $r_{f}=N_{c}^{f} g_{A}^{f} Q_{f}$. The sum runs over all charged SM fermions $f$, with $m_{f}$ the SM fermion masses. Furthermore, $g_{A}^{f}=g T_{3}^{f} /\left(2 \cos \theta_{W}\right)$ is the $Z$-boson axial coupling to SM fermions, with $g$ the weak coupling, $\theta_{W}$ the Weinberg angle, and $T_{f}^{3}(= \pm 1 / 2)$ the eigenvalue of the third component of weak isospin, $N_{c}^{f}=3(1)$ for quarks (leptons), and $Q_{f}$ is the electric charge in units of the elementary charge $e$. The $B_{f}$ and $C_{f}$ terms are defined as

$$
\begin{aligned}
& B_{f} \equiv \operatorname{Disc}\left[B_{0}\left(M_{Z}^{2}, m_{f}, m_{f}\right)\right], \\
& C_{f} \equiv C_{0}\left(0,0, M_{Z}^{2}, m_{f}, m_{f}, m_{f}\right),
\end{aligned}
$$

with $B_{0}$ and $C_{0}$ the scalar two- and three-point PassarinoVeltman functions, respectively (see Ref. [13] for their explicit expressions), and $\operatorname{Disc}\left[B_{0}\right]$ the discontinuity of the function. They are both finite functions that can be evaluated numerically, for example, by PACKAGE X [14].

Then, one has

$$
\frac{1}{3} \sum_{\mathrm{pol}} \mathcal{M}_{M} \mathcal{M}_{M}^{\dagger}=\frac{2}{3} \frac{\alpha_{D} \alpha}{\pi} M_{Z}^{2}\left|C_{M}\right|^{2}
$$

where $\alpha_{D}=e_{D}^{2} / 4 \pi$ and $\alpha=e^{2} / 4 \pi$ are the fine structure constants, and $C_{M}=\sum_{f} d_{M}^{f} \xi^{f}\left(m_{f}\right)$, where

$$
\xi^{f}\left(m_{f}\right) \equiv \frac{r_{f} m_{f}}{\Lambda}\left(3+B_{f}+2 m_{f}^{2} C_{f}\right) .
$$

The $C P$-violating contribution to the on-shell amplitude induced by the electric-dipole moment in Eq. (4) is given by

$$
\mathcal{M}_{E}=i \frac{e_{D} e}{4 \pi^{2}} C_{E}\left(k_{1}^{\mu}-k_{2}^{\mu}\right) g^{\alpha \beta} \epsilon_{\mu}^{Z}(q) \epsilon_{\alpha}\left(k_{1}\right) \bar{\epsilon}_{\beta}\left(k_{2}\right) .
$$

Accordingly, we find that

$$
\frac{1}{3} \sum_{\mathrm{pol}} \mathcal{M}_{E} \mathcal{M}_{E}^{\dagger}=\frac{2}{3} \frac{\alpha_{D} \alpha}{\pi} M_{Z}^{2}\left|C_{E}\right|^{2},
$$

where $C_{E}=\sum_{f} d_{E}^{f} \xi^{f}\left(m_{f}\right)$.

The amplitudes in Eq. (8) and Eq. (16) are both proportional to the $Z$-boson axial coupling $g_{A}$. In the limit 
of restored $S U(2)$ symmetry, both squared amplitudes go to zero as $M_{Z} \rightarrow 0$.

In the on-shell amplitude, all polarization vectors satisfy the transversality condition, namely, $\epsilon_{\mu}(k) k^{\mu}=0$, with $\epsilon_{\mu}$ a generic polarization vector. One can verify that the amplitudes in Eq. (8) and Eq. (16) satisfy the Ward identities by substituting the polarizations $\epsilon_{\alpha}\left(k_{1}\right)$ and $\epsilon_{\beta}\left(k_{2}\right)$ with the corresponding momenta.

For the $C P$-conserving part, the Ward identity for the $Z$ boson —obtained by substituting $\epsilon_{\mu}^{Z}(q)$ with $q_{\mu}$-requires a [SU(2) invariant] counterterm $H H^{\dagger} F_{\mu \nu} \bar{F}^{\mu \nu}$ in the effective theory because of the divergence generated by the insertion of the dipole operator in the diagram where the $Z$ Goldstone boson decays. This term does not affect our computation.

Lagrangians. - It is useful to see how the above amplitudes can be derived from a manifestly gauge invariant Lagrangian in configuration space. In particular, for the Lagrangian induced by the magnetic-dipole moment, we have

$$
\mathcal{L}_{\text {eff }}^{(M)}=\frac{e_{D} e}{4 \pi^{2} M_{Z}^{2}} \sum_{i=1}^{3} \bar{C}_{i} \mathcal{O}_{i}(x)
$$

where the dimension-six operators $\mathcal{O}_{i}$ are given by

$$
\begin{aligned}
& \mathcal{O}_{1}(x)=Z_{\mu \nu} \tilde{B}^{\mu \alpha} A_{\alpha}^{\nu}, \\
& \mathcal{O}_{2}(x)=Z_{\mu \nu} B^{\mu \alpha} \tilde{A}_{\alpha}^{\nu}, \\
& \mathcal{O}_{3}(x)=\tilde{Z}_{\mu \nu} B^{\mu \alpha} A_{\alpha}^{\nu} .
\end{aligned}
$$

The field strengths $F_{\mu \nu} \equiv \partial_{\mu} F_{\nu}-\partial_{\nu} F_{\mu}$, for $F_{\mu \nu}=$ $(Z, B, A)_{\mu \nu}$, correspond to the $Z$ boson $\left(Z_{\mu}\right)$, dark photon $\left(B_{\mu}\right)$, and photon $\left(A_{\mu}\right)$ fields, respectively, and $\tilde{F}^{\mu \nu} \equiv$ $\varepsilon^{\mu \nu \alpha \beta} F_{\alpha \beta}$ is the dual field strength. Matching the on-shell amplitude for the $Z \rightarrow \gamma \bar{\gamma}$ process-as obtained by using the effective Lagrangian in Eq. (18)—with the corresponding one in Eq. (8), yields

$$
\begin{aligned}
& \bar{C}_{1}=-\sum_{f} \frac{r_{f} m_{f} d_{M}^{f}}{\Lambda}\left(5+2 B_{f}+2 C_{f}\left(m_{f}^{2}+M_{Z}^{2}\right)\right), \\
& \bar{C}_{2}=-3 \sum_{f} \frac{r_{f} m_{f} d_{M}^{f}}{\Lambda}\left(2+B_{f}\right), \\
& \bar{C}_{3}=2 \sum_{f} \frac{r_{f} m_{f} d_{M}^{f}}{\Lambda}\left(4+2 B_{f}+C_{f} M_{Z}^{2}\right) .
\end{aligned}
$$

The Lagrangian induced by the electric-dipole moment is instead

$$
\mathcal{L}_{\text {eff }}^{(E)}=\frac{e_{D} e}{4 \pi^{2} M_{Z}^{2}} C_{E} \mathcal{O}(x)
$$

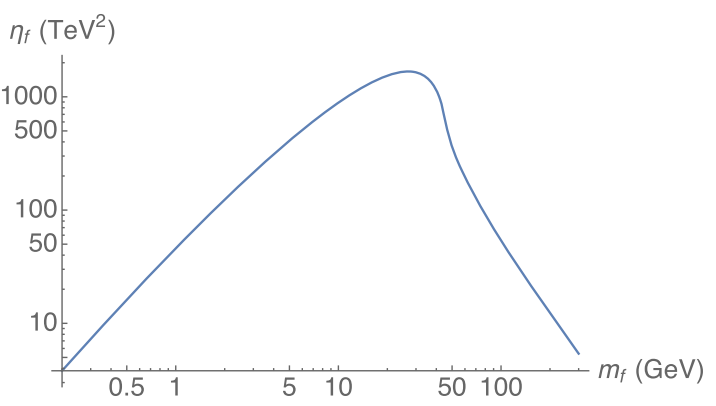

FIG. 3. Double logarithmic plot of the function $\eta_{f}\left(m_{f}\right)=$ $\left|\xi^{f}\left(m_{f}\right)\right|^{2}(\Lambda / \mathrm{TeV})^{2} / r_{f}^{2}$ containing the loop fermion-mass dependence characterizing a single-fermion contribution to $\Gamma(Z \rightarrow \gamma \bar{\gamma})$ in Eq. (25). The nontrivial behavior around $m_{f} \sim 30 \mathrm{GeV}$ explains the flavor dependence of $X_{f}$ in Table I.

where $C_{E}$ is given below Eq. (17) and the dimension-six operator is

$$
\mathcal{O}(x)=Z_{\mu \nu} A^{\mu \alpha} B_{\alpha}^{\nu} .
$$

The local operators entering the Lagrangians in Eqs. (18) and (23) are both $C$ odd. The operators in Eq. (18) are also $P$ odd, and therefore overall $C P$ even. The operator in Eq. (23) is $P$ even, and therefore overall $C P$ odd. The corresponding $C P$ properties are hence as expected for being induced by a magnetic- and electric-dipole operator, respectively.

The Lagrangians in Eq. (18) and Eq. (23) are different from those (induced by anomalies) studied in Refs. [15] or [16], from which the Landau-Yang theorem is bypassed by having massive final states.

Decay rate. - The total $Z \rightarrow \gamma \bar{\gamma}$ decay width is obtained from Eq. (14) and Eq. (17), and is given by

$$
\Gamma(Z \rightarrow \gamma \bar{\gamma})=\frac{\alpha_{D} \alpha M_{Z}}{24 \pi^{3}}\left(\left|C_{M}\right|^{2}+\left|C_{E}\right|^{2}\right),
$$

where $C_{M, E}=\sum_{f} d_{M, E}^{f} \xi^{f}\left(m_{f}\right)$, and $\xi^{f}\left(m_{f}\right)$ is given in Eq. (15). In Fig. 3, the function $\eta_{f}\left(m_{f}\right)=\left|\xi^{f}\left(m_{f}\right)\right|^{2} \times$ $(\Lambda / \mathrm{TeV})^{2} / r_{f}^{2}$ [pinpointing the $m_{f}$ dependence of a single $f$ SM-fermion contribution to $\Gamma(Z \rightarrow \gamma \bar{\gamma})]$ is plotted versus the fermion mass.

The branching ratios $\mathrm{BR}_{f}=\Gamma_{f} / \Gamma_{Z}$, which by definition assume a single SM-fermion contribution from flavor $f$ to

TABLE I. BRs for the largest single-fermion $Z \gamma \bar{\gamma}$-loop contributions. The BR corresponding to each SM fermion of flavor $f$ would be $\mathrm{BR}_{f}=\alpha_{D}\left(\left|d_{M}^{f}\right|^{2}+\left|d_{E}^{f}\right|^{2}\right) /(\Lambda / \mathrm{TeV})^{2} X_{f}$. The $C P$-conserving and $C P$-odd parts are equal.

\begin{tabular}{cccccccc}
\hline \hline & $b$ & $t$ & $s$ & $c$ & $\tau$ & $\mu$ & \\
\hline$X_{f}$ & 4.80 & 0.82 & 0.014 & 4.78 & 1.30 & 0.017 & $\times 10^{-9}$ \\
\hline \hline
\end{tabular}


Eq. (25), can be obtained from Table I, with $\mathrm{BR}_{f}=$ $\alpha_{D}\left(\left|d_{M}^{f}\right|^{2}+\left|d_{E}^{f}\right|^{2}\right) /(\Lambda / \mathrm{TeV})^{2} X_{f}$. For lighter flavors $(u, d, e)$-whose coefficients $d_{M}^{f} / \Lambda$ and $d_{E}^{f} / \Lambda$ are strongly constrained by astrophysics [11] $-\mathrm{BR}_{f}$ turns out to be further suppressed by the loop $m_{f}$ dependence.

Assuming universal magnetic- and electric-dipole moments, $d_{M}$ and $d_{E}$, by summing up all fermion contributions (including interferences) for all heavier flavors in Table I, we obtain

$\mathrm{BR}(Z \rightarrow \gamma \bar{\gamma}) \simeq \frac{2.52 \alpha_{D}}{(\Lambda / \mathrm{TeV})^{2}}\left(\left|d_{M}\right|^{2}+\left|d_{E}\right|^{2}\right) \times 10^{-8}$.

The resulting $\operatorname{BR}(Z \rightarrow \gamma \bar{\gamma})$ is quite suppressed, resulting from an effective two-loop computation, also featuring a few partial cancellations. Depending on the values assumed for the dipole moments in Eq. (5), the dark-sector energy scale $\Lambda$, and coupling $\alpha_{D}$, this channel could be observable at colliders collecting large samples of $Z$ bosons.

If we assume that the dipole momenta in Eq. (5) are produced at the limit for perturbative interactions in the dark sector, where $g_{L}^{f} g_{R}^{f *} \simeq(4 \pi)^{2}$-we can take $d_{E} \simeq d_{M} \simeq 1 / 2$. In this case, assuming $\alpha_{D}$ of order 0.1 and $\Lambda$ around $1 \mathrm{TeV}$, one would get $\operatorname{BR}(Z \rightarrow \gamma \bar{\gamma}) \simeq 10^{-9}$ from Eq. (26). Smaller (and perhaps more realistic) couplings, like $d_{E} \simeq d_{M} \simeq 0.1$, would anyhow give $\operatorname{BR}(Z \rightarrow \gamma \bar{\gamma}) \simeq 4 \times 10^{-11}$, for the same $\alpha_{D}$ and $\Lambda$.

Our prediction stems from a weakly coupled UV complete model. One could also envisage different frameworks with effective couplings generated, for instance, by a nonperturbative dynamics, pushing the effecting $\Lambda$ scale to lower values, and correspondingly enhancing $\operatorname{BR}(Z \rightarrow \gamma \bar{\gamma})$, and possibly saturating the present LEP limit of BR $<10^{-6}$ on $Z$ monophoton decays [9].

Note that while the processes involving the dark-photon coupling to the Higgs in Ref. [5] are mainly ruled by the $\alpha_{D}$ magnitude, the present $Z$-decay rate, similarly to the FCNC decays in Ref. [6], is governed also by the $\Lambda$ scale parameter. The strongest bounds on the model mass spectrum, and therefore on $\Lambda$, come from messenger-pair production at the LHC. These (taking into account the possibility of different signatures) are similar to the current squarks and sleptons bounds [17].

Experimental perspectives.-Large samples of $Z$ bosons produced in high-energy collisions will be needed to test the prediction in Eq. (26). Present and future hadron colliders will collect a very large number of $Z$ 's. On the other hand, separating the background for a final state involving moderate missing momentum (like the typical final state in $Z \rightarrow \gamma \bar{\gamma}$ ) would be in general very challenging in proton collisions. One could try to focus on boosted $Z$ systems, which anyhow would deplete the statistics. The cross section for $Z$-boson production at the LHC is about $59 \mathrm{nb}$ (at $13 \mathrm{TeV}$ ) [18], which, with an integrated luminosity of $300 \mathrm{fb}^{-1}$, would provide about $2 \times 10^{10}$
$Z$ bosons. Ten times more $Z$ 's will be produced with the integrated luminosity of $3 \mathrm{ab}^{-1}$ expected at the HL-LHC. Assuming the dipole momenta in Eq. (5) at the limit for perturbative interactions in the dark sector, for $\alpha_{D} \simeq 0.1$ and $\Lambda \simeq 1 \mathrm{TeV}$, corresponding to $\operatorname{BR}(Z \rightarrow \gamma \bar{\gamma}) \simeq 10^{-9}$, one could have about $200 Z \rightarrow \gamma \bar{\gamma}$ events at the HL-LHC. A more favorable sample (with a less favorable environment) is expected at a $100 \mathrm{TeV}$ collider-where the production cross section for the $Z$ boson is about $0.4 \mu \mathrm{b}$ [19], with the number of produced $Z$ 's around $10^{13}$ for a luminosity of $30 \mathrm{ab}^{-1}$, and $10^{4} Z \rightarrow \gamma \bar{\gamma}$ decays.

The study of the final-state angular distribution for polarized $Z$ bosons would allow us to distinguish the dark photon from the axion case [16], the two having otherwise similar signatures.

The best opportunity to study the $Z \rightarrow \gamma \bar{\gamma}$ decay channel-and possibly discover a dark photon-would come from the clean environment of the Future Circular Collider (FCC-ee), with its projected production of $10^{13}$ $Z$ bosons [20].

[1] L. D. Landau, Dokl. Akad. Nauk Ser. Fiz. 60, 207 (1948); C. N. Yang, Phys. Rev. 77, 242 (1950).

[2] R. Essig et al., arXiv:1311.0029; M. Raggi and V. Kozhuharov, Riv. Nuovo Cimento 38, 449 (2015); J. Alexander et al., arXiv:1608.08632; F. Curciarello, EPJ Web Conf. 118, 01008 (2016); A. Denig, EPJ Web Conf. 130, 01005 (2016).

[3] B. Holdom, Phys. Lett. 166B, 196 (1986); F. del Aguila, M. Masip, and M. Perez-Victoria, Nucl. Phys. B456, 531 (1995).

[4] B. A. Dobrescu, Phys. Rev. Lett. 94, 151802 (2005).

[5] E. Gabrielli, M. Heikinheimo, B. Mele, and M. Raidal, Phys. Rev. D 90, 055032 (2014); S. Biswas, E. Gabrielli, M. Heikinheimo, and B. Mele, J. High Energy Phys. 06 (2015) 102; Phys. Rev. D 93, 093011 (2016); 96, 055012 (2017).

[6] E. Gabrielli, B. Mele, M. Raidal, and E. Venturini, Phys. Rev. D 94, 115013 (2016).

[7] M. Fabbrichesi, E. Gabrielli, and B. Mele, Phys. Rev. Lett. 119, 031801 (2017).

[8] B. A. Gradwohl and J. A. Frieman, Astrophys. J. 398, 407 (1992); E. D. Carlson, M. E. Machacek, and L. J. Hall, Astrophys. J. 398, 43 (1992); R. Foot, Int. J. Mod. Phys. D 13, 2161 (2004); L. Ackerman, M. R. Buckley, S. M. Carroll, and M. Kamionkowski, Phys. Rev. D 79, 023519 (2009); J. Fan, A. Katz, L. Randall, and M. Reece, Phys. Rev. Lett. 110, 211302 (2013); R. Foot and S. Vagnozzi, Phys. Rev. D 91, 023512 (2015); M. Heikinheimo, M. Raidal, C. Spethmann, and H. Veerme, Phys. Lett. B 749, 236 (2015); P. Agrawal, F. Y. Cyr-Racine, L. Randall, and J. Scholtz, J. Cosmol. Astropart. Phys. 05 (2017) 022.

[9] M. Acciarri et al. (L3 Collaboration), Phys. Lett. B 412, 201 (1997); O. Adriani et al. (L3 Collaboration), Phys. Lett. B 297, 469 (1992); P. Abreu et al. (DELPHI Collaboration), Z. Phys. C 74, 577 (1997); R. Akers et al. (OPAL Collaboration), Z. Phys. C 65, 47 (1995). 
[10] E. Gabrielli and M. Raidal, Phys. Rev. D 89, 015008 (2014); E. Gabrielli, L. Marzola, and M. Raidal, Phys. Rev. D 95, 035005 (2017).

[11] S. Hoffmann, Phys. Lett. B 193, 117 (1987); M. Giannotti, I. Irastorza, J. Redondo, and A. Ringwald, J. Cosmol. Astropart. Phys. 05 (2016) 057.

[12] S. L. Adler, Phys. Rev. 177, 2426 (1969).

[13] G. Passarino and M. J. G. Veltman, Nucl. Phys. B160, 151 (1979).

[14] H. H. Patel, Comput. Phys. Commun. 197, 276 (2015); 218, 66 (2017).

[15] W. Y. Keung, I. Low, and J. Shu, Phys. Rev. Lett. 101, 091802 (2008).
[16] J. A. Dror, R. Lasenby, and M. Pospelov, Phys. Rev. Lett. 119, 141803 (2017); Phys. Rev. D 96, 075036 (2017).

[17] C. Patrignani et al. (Particle Data Group), Chin. Phys. C 40, 100001 (2016).

[18] G. Aad et al. (ATLAS Collaboration), Phys. Lett. B 759, 601 (2016).

[19] N. Arkani-Hamed, T. Han, M. Mangano, and L. T. Wang, Phys. Rep. 652, 1 (2016).

[20] M. Bicer et al. (TLEP Design Study Working Group), J. High Energy Phys. 01 (2014) 164; David d'Enterria, arXiv:1602.05043. 